\title{
ON MEAN DENSITIES OF INHOMOGENEOUS GEOMETRIC PROCESSES ARISING IN MATERIAL SCIENCE AND MEDICINE
}

\author{
VinCENZO CAPASSO AND ELENA VILLA
}

Dept. of Mathematics, University of Milan, Via Saldini 50, 20133, Milano, Italy

e-mail: vincenzo.capasso@unimi.it, elena.villa@mat.unimi.it

(Accepted January 12, 2007)

\begin{abstract}
The scope of this paper is to offer an overview of the main results obtained by the authors in recent literature in connection with the introduction of a Delta formalism, á la Dirac-Schwartz, for random generalized functions (distributions) associated with random closed sets, having an integer Hausdorff dimension $n$ lower than the full dimension $d$ of the environment space $\mathbb{R}^{d}$. A concept of absolute continuity of random closed sets arises in a natural way in terms of the absolute continuity of suitable mean content measures, with respect to the usual Lebesgue measure on $\mathbb{R}^{d}$. Correspondingly mean geometric densities are introduced with respect to the usual Lebesgue measure; approximating sequences are provided, that are of interest for the estimation of mean geometric densities of lower dimensional random sets such as fibre processes, surface processes, etc. Many models in material science and in biomedicine include time evolution of random closed sets, describing birthand-growth processes; the Delta formalism provides a natural framework for deriving evolution equations for mean densities at all (integer) Hausdorff dimensions, in terms of the relevant kinetic parameters.
\end{abstract}

Keywords: approximation of geometric densities, birth-and-growth processes, geometric densities, geometric measure theory, random distributions, random measures, stochastic geometry.

\section{INTRODUCTION}

Many real phenomena may be modelled as random closed sets in $\mathbb{R}^{d}$, and in several situations as evolving random closed sets. Application areas include crystallization processes, Figs. 1 and 6 (see
Capasso, 2003; Capasso and Micheletti, 2005 and the references therein; see also Ubukata, 2003 for the crystallization processes on sea shells, Fig. 3); tumor growth (Anderson, 2003) and angiogenesis, Figs. 2 and 4 (Carmeliet and Jain, 2000); spread of fires in the woods, spread of a pollutant in an environment; etc.

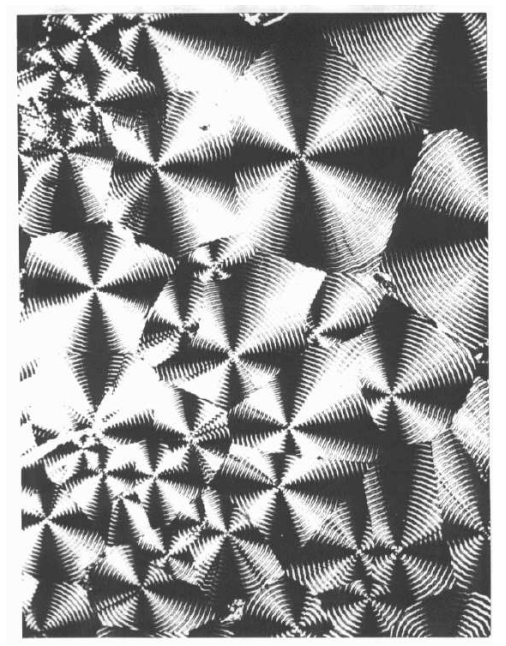

(a) real

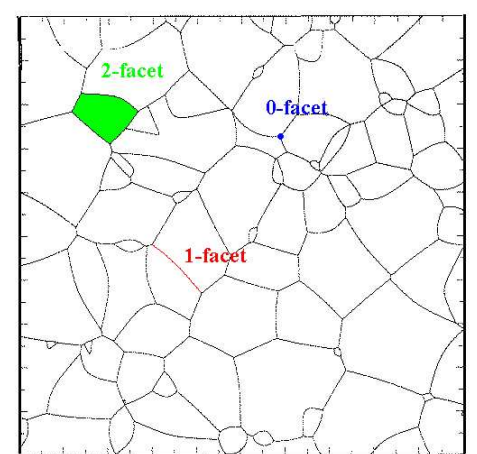

(b) simulated

Fig. 1. The final tessellation in a real experiment of a crystallization process of a polymer. This picture, together with the simulated one, shows the relevance of components at all integer Hausdorff dimensions for describing the final morphology. Reproduced by permission of BASELL Polyolefins, "G. Natta” Research Centre, Ferrara, Italy. 


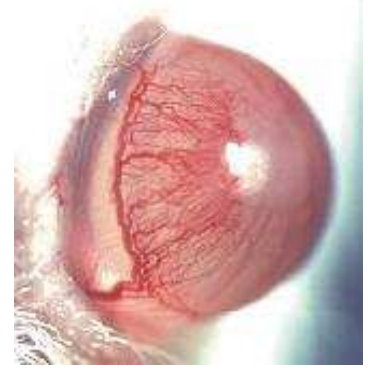

(a)

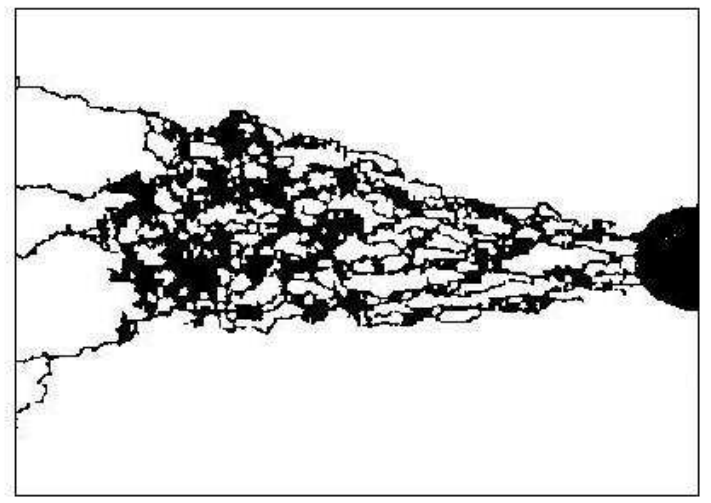

(b)

Fig. 2. (a) Angiogenesis on a rat cornea. Reproduced by permission from Corada et al. (2002). (b) A simulation of an angiogenesis due to a localized tumor mass (black region on the right). Reproduced by permission from Chaplain and Anderson (1999).

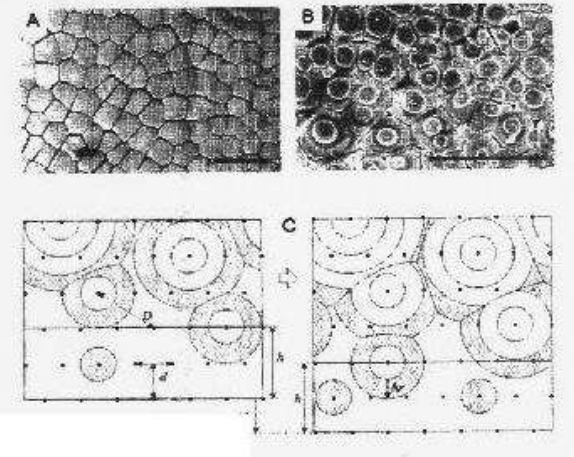

Fig. 3. Crystallization on the shell of a bivalve. $A, B$ real, and $C$ simulated, of the relevant birth-and-growth mechanism. Reproduced by permission from Ubukata (2003).

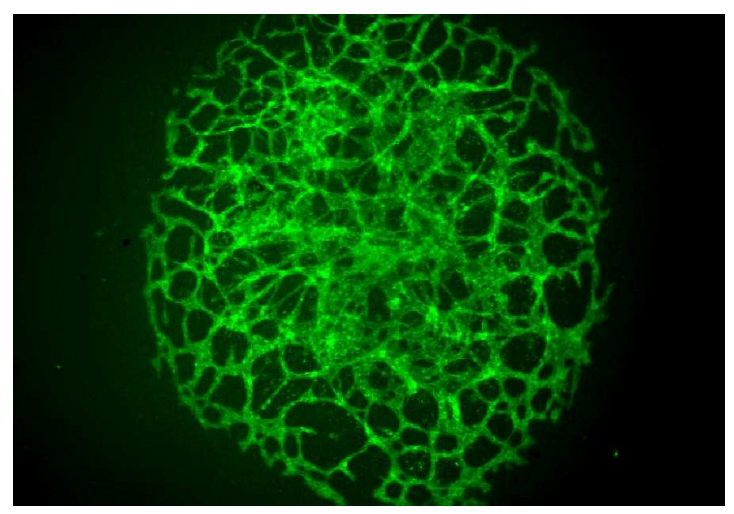

Fig. 4. Vascularization of an allantoid. An important example of a fibre process of Hausdorff dimension 1 in a 3D space. Reproduced by permission from Crosby et al. (2005).
All quoted processes may be described by time dependent random closed sets at different Hausdorff dimensions (for instance, crystallization processes are modelled in general by full dimensional growing sets, and lower dimensional interfaces, while angiogenesis by systems of random curves). In particular, because of the coupling with a suitable underlying field, such as temperature, nutrients, etc., all these kinds of phenomena may be modelled as space-time structured stochastic birth-and-growth processes in a $d$-dimensional space $\mathbb{R}^{d}$ driven by a spatially marked point process $N=\left\{\left(T_{i}, X_{i}\right)\right\}_{i \in \mathbb{N}}$, modelling births, at random times $T_{i} \in \mathbb{R}_{+}$, and related random spatial locations $X_{i} \in \mathbb{R}^{d}$ (Johnson and Mehl, 1939; Stoyan et al., 1995). Once born, each germ generates a grain subject to surface growth, with a speed $G(t, x)>$ 0 which should, in general, be assumed spacetime dependent. As a first approximation one may assume that the kinetic parameters of the process are given deterministic (space and time dependent) fields, obtained via a homogenization at the scale of the underlying field.

We remind that a random closed set $\Xi$ in $\mathbb{R}^{d}$ is a measurable map

$$
\Xi:(\Omega, \mathscr{F}, \mathbb{P}) \longrightarrow\left(\mathbb{F}, \sigma_{\mathbb{F}}\right)
$$

(i.e., $\Xi(S) \in \mathscr{F}$ for all $S \in \sigma_{\mathbb{F}}$ ), where $\mathbb{F}$ denotes the class of the closed subsets in $\mathbb{R}^{d}$, and $\sigma_{\mathbb{F}}$ is the $\sigma$ algebra generated by the so called hit-or-miss topology (see Matheron, 1975).

In several real applications it is of interest to study random closed sets of different Hausdorff dimensions. 
A random tessellation has been characterized by its interfaces; dimension 0 for vertices, 1 for edges, etc. (see Fig. 1, and, e.g., Møller, 1992; 1994).

For definitions and basic properties of Hausdorff measure and Hausdorff dimension see, e.g., Falconer (1985); Federer (1996); Ambrosio et al. (2000). Denoting by $\mathscr{H}^{n}$ the $n$-dimensional Hausdorff measure, we recall that $\mathscr{H}^{0}$ is the usual counting measure; for any Borel set $B \subset \mathbb{R}, \mathscr{H}^{d}(B)$ coincides with the usual $d$-dimensional Lebesgue measure of $B$; for $1 \leq n<d$ integer, $\mathscr{H}^{n}(B)$ coincides with the classical $n$-dimensional measure of $B$ if $B \in \mathscr{B}_{\mathbb{R}^{d}}$ (the Borel $\sigma$-algebra of $\mathbb{R}^{d}$ ) is contained in a $C^{1} n$ dimensional manifold embedded in $\mathbb{R}^{d}$. Further, we recall that the Hausdorff dimension of a set $A \subset \mathbb{R}^{d}$ is defined as

$$
\operatorname{dim}_{\mathscr{H}}(A):=\inf \left\{0 \leq s<\infty \mid \mathscr{H}^{s}(A)=0\right\},
$$

so that, in particular, a point has Hausdorff dimension 0 , a curve (or a fibre) has Hausdorff dimension 1, a hypersurface in $\mathbb{R}^{d}$ has Hausdorff dimension $d-1$. Note that the Hausdorff dimension of a set $A$ need not to be an integer (e.g., consider the Cantor set), and $\operatorname{dim}_{\mathscr{H}}(A)=s$ does not imply that $\mathscr{H}^{s}(A)$ is positive and finite (we may have $\operatorname{dim}_{\mathscr{H}}(A)=s$ and $\mathscr{H}^{s}(A)=0$, or $\left.\mathscr{H}^{s}(A)=\infty\right)$.

We say that a random closed set $\Theta$ has Hausdorff dimension $n$ if $\operatorname{dim}_{\mathscr{H}} \Theta(\omega)=n$ for a.e. $\omega \in \Omega$ ); in such case we also write $\Theta_{n}$ to remind its Hausdorff dimension.

In dependence of its regularity, a random closed set $\Theta_{n}$ with Hausdorff dimension $n$ may induce a random Radon measure

$$
\mu_{\Theta_{n}}(\cdot):=\mathscr{H}^{n}\left(\Theta_{n} \cap \cdot\right)
$$

on $\mathbb{R}^{d}$, and, as a consequence, an expected measure

$$
\mathbb{E}\left[\mu_{\Theta_{n}}\right](\cdot):=\mathbb{E}\left[\mathscr{H}^{n}\left(\Theta_{n} \cap \cdot\right)\right] .
$$

(For a discussion about measurability of $\mathscr{H}^{n}\left(\Theta_{n}\right)$ we refer to Zahle, 1982; Baddeley and Molchanov, 1997).

A case of interest in applications (Møller, 1992; Hahn et al., 1999; Beneš and Rataj, 2004) is the one in which the mean measure admits a density (a classical function) with respect to the usual Lebesgue measure $v^{d}$ in $\mathbb{R}^{d}$; for $n=d$ it is known as degree of crystallinity (Avrami, 1939; Kolmogorov, 1937); for any other integer $0 \leq n \leq d-1$ it is known as $n$-facet density; for fibre processes $(n=1)$ we speak of fibre density, etc. (see Fig. 5).

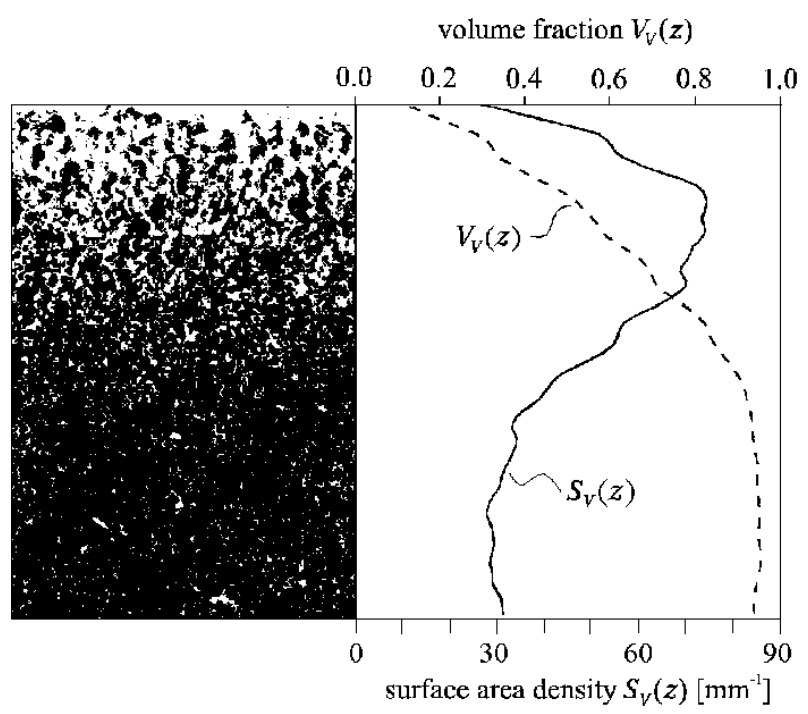

Fig. 5. Degree of crystallinity $\left(V_{v}(z)\right)$ and surface density $\left(S_{V}(z)\right)$ for an experimental $2 D$ sample of tungsten-copper alloy. Reproduced by permission from Hahn et al. (1999).

We wish to stress the fact that it is not always true that the mean measure $\mathbb{E}\left[\mu_{\Theta}\right]$ admits a classical function as density, so that the need of introducing a concept of absolute continuity for random closed sets arises in a natural way. Thus, it is of interest to distinguish between random closed sets which induce an absolutely continuous expected measure, and random closed sets which induce a singular one. To this aim we introduce definitions of discrete, continuous and absolutely continuous random closed set, coherently with the classical 0dimensional case, in order to propose an extension of the standard definition of discrete, continuous, and absolutely continuous random variable, respectively. (For preliminary definitions and results see also Capasso and Villa, 2006b;c.)

Our approach as reviewed here from a series of papers in the subject (Ambrosio et al., 2006; Capasso and Villa, 2006d; 2007) is based on a theory of random distributions as generalized densities of random measures, and mean geometric densities as expected values of random generalized densities. In particular we introduce a Delta formalism, á la Dirac-Schwartz, for the description of random measures associated with random closed sets of lower dimensions, such that the well known usual Dirac delta at a point follows as a particular case (see, for instance, Kolmogorov and Fomin, 1970; Vladimirov, 1979; Jones, 1982). For a first attempt to deal with random distributions we refer to Matheron (1965).

In this way, in the context of time dependent growing random sets, we provide a natural framework for deriving evolution equations for mean densities 
at all (integer) Hausdorff dimensions, in terms of the relevant kinetic parameters associated with a given growth process (Capasso and Villa, 2006d).

As an important example modelling a variety of real phenomena we discuss later the case of birthand-growth processes, subject to a normal growth model for the grains. We evidence the case in which such mean geometric densities are classical functions, which happens in the case of absolutely continuous random sets.

Hence we show how evolution equations for some relevant mean densities take advantage of the "localizing" action of the Delta formalism for writing evolution equations in terms of the relevant local (in time and space) kinetic parameters of the system.

Finally, as for the usual Dirac delta as a generalized function, we show how to express a mean geometric density $\mathbb{E}\left[\delta_{\Theta_{n}}\right]$ in terms of a suitable approximating sequence of classical functions. This turns out of great importance in several real applications, whenever we wish to estimate the density of the mean measure $\mathbb{E}\left[\mu_{\Theta_{n}}\right]$. For example, when $n=1$ as in the case of a fibre process, or a line process, or when $n=d-1$ as in the case of a surface process (Ambrosio et al., 2006).

\section{GENERALIZED DENSITIES}

In the sequel we will refer to a class of sufficiently regular closed sets in the Euclidean space $\mathbb{R}^{d}$, of integer dimension $n$. We denote by $B_{r}(x)$ the ball with center $x$ and radius $r$.

Definition 1 ( $n$-regular set) Given an integer $n \in$ $[0, d]$, we say that a closed subset $S$ of $\mathbb{R}^{d}$ is $n$-regular, if it satisfies the following conditions:

(i) $\mathscr{H}^{n}\left(S \cap B_{R}(0)\right)<\infty \quad$ for any $R>0$;

(ii) $\lim _{r \rightarrow 0} \frac{\mathscr{H}^{n}\left(S \cap B_{r}(x)\right)}{b_{n} r^{n}}=1$ for $\mathscr{H}^{n}-$ a.e. $x \in S$.

Here $b_{n}$ is the volume of the unit ball in $\mathbb{R}^{n}$.

Remark 2 Note that condition (ii) is related to a characterization of the $\mathscr{H}^{n}$-rectifiability of the set $S$ (Falconer, 1985, p. 256, 267; Ambrosio et al., 2000, p. 83).

We may observe that if $A_{n}$ is an $n$-regular closed set in $\mathbb{R}^{d}$, we have

$$
\lim _{r \rightarrow 0} \frac{\mathscr{H}^{n}\left(A_{n} \cap B_{r}(x)\right)}{b_{n} r^{n}}= \begin{cases}1 & \mathscr{H}^{n} \text {-a.e. } x \in A_{n}, \\ 0 & \forall x \notin A_{n} .\end{cases}
$$

As a consequence (by assuming $0 \cdot \infty=0$ ), for $0 \leq n<$ $d$ we have

$$
\begin{aligned}
\lim _{r \rightarrow 0} \frac{\mathscr{H}^{n}\left(A_{n} \cap B_{r}(x)\right)}{b_{d} r^{d}} & =\lim _{r \rightarrow 0} \frac{\mathscr{H}^{n}\left(A_{n} \cap B_{r}(x)\right)}{b_{n} r^{n}} \frac{b_{n} r^{n}}{b_{d} r^{d}} \\
& =\left\{\begin{array}{cl}
\infty & \mathscr{H}^{n} \text {-a.e. } x \in A_{n}, \\
0 & \forall x \notin A_{n} .
\end{array}\right.
\end{aligned}
$$

It is known that every positive Radon measure $\mu$ on $\mathbb{R}^{d}$ can be represented in the form

$$
\mu=\mu_{\ll}+\mu_{\perp},
$$

where $\mu_{\ll}$ and $\mu_{\perp}$ are the absolutely continuous part with respect to $v^{d}$, and the singular part of $\mu$, respectively. We may notice that, if $A_{n}$ is an $n$-regular closed set in $\mathbb{R}^{d}$ with $n<d$, then the measure

$$
\mu_{A_{n}}(\cdot):=\mathscr{H}^{n}\left(A_{n} \cap \cdot\right)
$$

is a singular measure with respect to $v^{d}$, and so the Radon-Nikodym derivative of $\mu_{A_{n} \ll}$ is zero $v^{d}$-a.e. $x \in \mathbb{R}^{d}$.

Even if, usually, by "density of $\mu$ " it is understood the Radon-Nikodym derivative of $\mu$ with respect to $v^{d}$, and so it is meant that $\mu$ is absolutely continuous, actually, it is well known in literature the Delta "function" at a point $X_{0}$, as the (generalized) density of the singular Dirac measure (Kolmogorov and Fomin, 1970). In analogy with the usual Dirac delta function $\delta_{x_{0}}(x)$ associated with a point $x_{0} \in \mathbb{R}^{d}$ (a 0-regular closed set), we may introduce the following definition.

Definition 3 (generalized density) We call $\delta_{A_{n}}$, the generalized density (or, briefly, the density) associated with $A_{n}$, the quantity

$$
\delta_{A_{n}}(x):=\lim _{r \rightarrow 0} \frac{\mathscr{H}^{n}\left(A_{n} \cap B_{r}(x)\right)}{b_{d} r^{d}}
$$

finite or not.

In this way $\delta_{A_{n}}(x)$ can be considered as the generalized density (or the generalized Radon-Nikodym derivative) of the measure $\mu_{A_{n}}$ with respect to the $d$-dimensional Lebesgue measure $v^{d}$.

Define the function

$$
\delta_{A_{n}}^{(r)}(x):=\frac{\mathscr{H}^{n}\left(A_{n} \cap B_{r}(x)\right)}{b_{d} r^{d}},
$$

and correspondingly the associated measure

$$
\mu_{A_{n}}^{(r)}(B):=\int_{B} \delta_{A_{n}}^{(r)}(x) \mathrm{d} x, \quad B \in \mathscr{B}_{\mathbb{R}^{d}} .
$$


With an abuse of notations, we may introduce the linear functionals $\delta_{A_{n}}^{(r)}$ and $\delta_{A_{n}}$ associated with the measures $\mu_{A_{n}}^{(r)}$ and $\mu_{A_{n}}$, respectively, as follows:

$$
\begin{aligned}
\left(\delta_{A_{n}}^{(r)}, f\right) & :=\int_{\mathbb{R}^{d}} f(x) \mu_{A_{n}}^{(r)}(\mathrm{d} x), \\
\left(\delta_{A_{n}}, f\right) & :=\int_{\mathbb{R}^{d}} f(x) \mu_{A_{n}}(\mathrm{~d} x),
\end{aligned}
$$

for any $f \in C_{c}\left(\mathbb{R}^{d}, \mathbb{R}\right)$, having denoted by $C_{c}\left(\mathbb{R}^{d}, \mathbb{R}\right)$ the space of all continuous functions from $\mathbb{R}^{d}$ to $\mathbb{R}$ with compact support.

It can be proved (see Capasso and Villa, 2006d) that the sequence of measures $\mu_{A_{n}}^{(r)}$ weakly* converges to the measure $\mu_{A_{n}}$; in other words, the sequence of linear functionals $\delta_{A_{n}}^{(r)}$ weakly* converges to the linear functional $\delta_{A_{n}}$, i.e., $\left(\delta_{A_{n}}, f\right) \rightarrow\left(\delta_{A}, f\right)$ for any $f \in$ $C_{c}\left(\mathbb{R}^{d}, \mathbb{R}\right)$.

Remark 4 In analogy with the classical Dirac delta, we may regard the continuous linear functional $\delta_{A_{n}}$ as a generalized function on the usual test space $C_{c}\left(\mathbb{R}^{d}, \mathbb{R}\right)$, and, in accordance with the usual representation of distributions in the theory of generalized functions, we formally write:

$$
\int_{\mathbb{R}^{d}} f(x) \delta_{A_{n}}(x) \mathrm{d} x:=\left(\delta_{A_{n}}, f\right) .
$$

Consider now random closed sets.

Definition 5 ( $n$-regular random set) Given an integer $n$, with $0 \leq n \leq d$, we say that a random closed set $\Theta_{n}$ in $\mathbb{R}^{d}$ is $n$-regular, if it satisfies the following conditions:

(i) for almost all $\omega \in \Omega, \Theta_{n}(\omega)$ is an n-regular set in $\mathbb{R}^{d}$

(ii) $\mathbb{E}\left[\mathscr{H}^{n}\left(\Theta_{n} \cap B_{R}(0)\right)\right]<\infty$ for any $R>0$.

If $\Theta_{n}$ is a random closed set in $\mathbb{R}^{d}$, the measure

$$
\mu_{\Theta_{n}}(\cdot):=\mathscr{H}^{n}\left(\Theta_{n} \cap \cdot\right)
$$

is a random measure, and consequently $\delta_{\Theta_{n}}$ is a random linear functional (i.e., $\left(\delta_{\Theta_{n}}, f\right)$ is a real random variable for any test function $f$ ).

By extending the definition of expected value of a random operator à la Pettis (or Gelfand-Pettis) (Araujo and Giné, 1980; Bosq, 2000), we may define the expected linear functional $\mathbb{E}\left[\delta_{\Theta_{n}}\right]$ associated with $\delta_{\Theta_{n}}$ as follows:

$$
\left(\mathbb{E}\left[\delta_{\Theta_{n}}\right], f\right):=\mathbb{E}\left[\left(\delta_{\Theta_{n}}, f\right)\right],
$$

and the mean generalized density $\mathbb{E}\left[\delta_{\Theta_{n}}\right](x)$ of $\mathbb{E}\left[\mu_{\Theta_{n}}\right]$ by the formal integral representation:

$$
\int_{A} \mathbb{E}\left[\delta_{\Theta_{n}}\right](x) \mathrm{d} x:=\mathbb{E}\left[\mathscr{H}^{n}\left(\Theta_{n} \cap A\right)\right],
$$

with

$$
\mathbb{E}\left[\delta_{\Theta_{n}}\right](x):=\lim _{r \rightarrow 0} \frac{\mathbb{E}\left[\mathscr{H}^{n}\left(\Theta_{n} \cap B_{r}(x)\right)\right]}{b_{d} r^{d}} .
$$

It can be shown (Capasso and Villa, 2006d) that an equivalent definition of (1) can be given in terms of the expected measure $\mathbb{E}\left[\mu_{\Theta_{n}}\right]$ by

$$
\left(\mathbb{E}\left[\delta_{\Theta_{n}}\right], f\right):=\int_{\mathbb{R}^{d}} f(x) \mathbb{E}\left[\mu_{\Theta_{n}}\right](\mathrm{d} x),
$$

for any $f$ such that the above integral makes sense.

By using the integral representation of $\left(\delta_{\Theta_{n}}, f\right)$ and $\left(\mathbb{E}\left[\delta_{\Theta_{n}}\right], f\right)$, Equation (1) becomes

$$
\int_{\mathbb{R}^{d}} f(x) \mathbb{E}\left[\delta_{\Theta_{n}}\right](x) \mathrm{d} x=\mathbb{E}\left[\int_{\mathbb{R}^{d}} f(x) \delta_{\Theta_{n}}(x) \mathrm{d} x\right] ;
$$

so that, formally, we may exchange integral and expectation.

Remark 6 When $n=d$, integral and expectation can be really exchanged by Fubini's theorem. Since in this case $\delta_{\Theta_{d}}(x)=\mathbf{1}_{\Theta_{d}}(x), v^{d}$-a.s., it follows that $\mathbb{E}\left[\delta_{\Theta_{d}}\right](x)=\mathbb{P}\left(x \in \Theta_{d}\right)$. In particular, in material science, the density $V_{V}(x):=\mathbb{P}\left(x \in \Theta_{d}\right)$ is known as the (degree of) crystallinity.

If $n=0$ and $\Theta_{0}=X_{0}$ is an absolutely continuous random point with pdf $p_{X_{0}}$, then $\mathbb{E}\left[\mathscr{H}^{0}\left(X_{0} \cap \cdot\right)\right]=$ $\mathbb{P}\left(X_{0} \in \cdot\right)$ is absolutely continuous, and its density $\mathbb{E}\left[\delta_{X_{0}}\right](x)$ is just the probability density function $p_{X_{0}}(x)$.

Thus, for any lower dimensional random closed set $\Theta_{n}$ in $\mathbb{R}^{d}$, while it is clear that $\mu_{\Theta_{n}(\omega)}$ is a singular measure, when we consider the expected measure $\mathbb{E}\left[\mu_{\Theta_{n}}\right]$, it may happen that it is absolutely continuous with respect to $v^{d}$, and so it may have a classical Radon-Nikodym derivative, so that $\mathbb{E}\left[\delta_{\Theta_{n}}\right](x)$ is a classical real-valued locally integrable function on $\mathbb{R}^{d}$ (see Capasso and Villa, 2006d, and Capasso and Villa, 2006b).

It is then of interest to say whether or not a classical mean density can be introduced for sets of lower Hausdorff dimensions, with respect to the usual Lebesgue measure on $\mathbb{R}^{d}$.

In order to respond to this further requirement, we propose a concept of absolute continuity for random closed sets. 


\section{DISCRETE, CONTINUOUS AND ABSOLUTELY CONTINUOUS RANDOM CLOSED SETS}

Definition 7 Let $\Theta$ be a random closed set in $\mathbb{R}^{d}$. We say that $\Theta$ is

- discrete if its probability law $\mathbb{P}_{\Theta}$ is concentrated on at most countable subset of $\mathbb{F}$; i.e there exist a family $\theta_{1}, \theta_{2}, \ldots$ of closed subsets of $\mathbb{R}^{d}$, and a family of real numbers $p_{1}, p_{2}, \ldots \in[0,1]$ such that $\mathbb{P}\left(\Theta=\theta_{i}\right)=p_{i}$ and $\sum_{i} p_{i}=1$;

- continuous if

$$
\mathbb{P}(\Theta=\theta)=0, \quad \forall \theta \in \mathbb{F} .
$$

Note that the definition given is consistent with the case in which $\Theta$ is a random variable or a random point in $\mathbb{R}^{d}$. In this case, since the possible realizations of $X$ are points in $\mathbb{R}^{d}$, then $\mathbb{P}(X=\theta)=0$ for every subset $\theta$ of $\mathbb{R}^{d}$ which is not a point, and so we say that $X$ is continuous if and only if $\mathbb{P}(X=x)=0$ for any $x \in \mathbb{R}^{d}$ (that is the usual definition).

Denoted by $\partial A$ the topological boundary of a set $A \subset \mathbb{R}^{d}$, in a large number of cases an equivalent condition to (2) is

$$
\mathbb{P}(\partial \Theta=\partial \theta)=0, \quad \forall \theta \in \mathbb{F},
$$

for instance when $\Theta$ is a random closed set in $\mathbb{R}^{d}$ with dimension less than $d$, or when $\Theta$ is a.s. the closure of its interior.

A definition of absolute continuity requires a reference measure; for a random point in $\mathbb{R}^{d}$ a definition of absolute continuity is given in terms of the absolute continuity of its law $P_{X}$ with respect to the usual Lebesgue measure on $\mathbb{R}^{d}$. So that for a random point $X$ we know that the following relations hold:

$$
\begin{aligned}
& X \text { absolutely continuous } \Rightarrow \begin{array}{l}
X \text { continuous, } \\
\text { but not the reverse; }
\end{array} \\
& X \text { discrete } \Rightarrow \quad X \text { singular, } \\
& \text { but not the reverse. }
\end{aligned}
$$

A random point is a particular random closed set of Hausdorff dimension 0; we now introduce a definition of absolute continuity for random closed sets, such that analogous relations for random sets hold with any Hausdorff dimension $n \in\{0,1, \ldots, d\}$; as a consequence (3) and (4) would follow as a particular case.

To avoid pathologies, as discussed later, we introduce now a class of random sets, which, in particular, include all random sets we are interested in the sequel.
Definition 8 ( $\mathscr{R}$ class) We say that a random closed set $\Theta$ in $\mathbb{R}^{d}$ belongs to the class $\mathscr{R}$ if

$\operatorname{dim}_{\mathscr{H}}(\partial \Theta)<d \quad$ and $\quad \mathbb{P}\left(\mathscr{H}^{\operatorname{dim}_{\mathscr{H}}(\partial \Theta)}(\partial \Theta)>0\right)=1$

Definition 9 (Strong absolute continuity) We say that a random closed set $\Theta$ is (strongly) absolutely continuous if $\Theta \in \mathscr{R}$ and

$$
\mathbb{E}\left[\mu_{\partial \Theta}\right] \ll v^{d}
$$

on $\mathscr{B}_{\mathbb{R}^{d}}$.

Notation: Without any further specification, in the following we will write "absolutely continuous random set" to mean a "strongly absolutely continuous random set".

Note that, if $\Theta \in \mathscr{R}$ with $\operatorname{dim}_{\mathscr{H}}(\Theta)=d$ is sufficiently regular so that $\operatorname{dim}_{\mathscr{H}}(\partial \Theta)=d-1$, then it is absolutely continuous if $\mathbb{E}\left[\mathscr{H}^{d-1}(\partial \Theta \cap \cdot)\right] \ll v^{d}(\cdot)$.

Remark 10 In the particular case that $\Theta=X$ is a random variable, Definition 9 coincides with the usual definition of absolute continuity of a random variable. In fact, $\operatorname{dim}_{\mathscr{H}} X=0, \partial X=X$, and $\mathbb{E}\left[\mathscr{H}^{0}(X)\right]=\mathbb{P}(X \in$ $\left.\mathbb{R}^{d}\right)=1$, so $X \in \mathscr{R}$ and then the condition (5) is equivalent to

$$
\mathbb{E}\left[\mathscr{H}^{0}(X \cap \cdot)\right]=\mathbb{P}(X \in \cdot) \ll v^{d}
$$

In analogy with measure theory, we give the following definition:

Definition 11 (singular random set) We say that a random closed set $\Theta \in \mathscr{R}$ is singular if and only if it is not absolutely continuous (in the sense of Definition 9).

In this way, the well known relations between the continuous and singular parts of a measure hold also for a random closed set, although in a different context.

Proposition 12 Let $\Theta \in \mathscr{R}$. Then

$$
\begin{aligned}
\Theta \text { absolutely continuous } \Rightarrow & \begin{array}{l}
\Theta \text { continuous, } \\
\text { but not the reverse; }
\end{array} \\
\Theta \text { discrete } \Rightarrow & \begin{array}{l}
\Theta \text { singular, } \\
\text { but not the reverse. }
\end{array}
\end{aligned}
$$


Proof. As a simple counterexample to (6) and (7), consider the random closed set $\Theta_{1}$ in $\mathbb{R}^{2}$ given by a random unit square with two edges on the $x$-axis, say $A$ and $B$, such that $A=(a, 0)$, where $a$ is a real valued random variable uniformly distributed in $[0,10]$ (consequently, $B=(a+1,0)$ ). It is clear that $\Theta_{1}$ is a continuous and singular random closed set.

Let us prove the first implication; the second one follows in a similar way.

By contradiction, let $\Theta$ be not continuous; then there exists $\theta \subset \mathbb{R}^{d}$ such that $\mathbb{P}(\Theta=\theta)>0$. Since $\Theta \in \mathscr{R}$, it follows that $\mathscr{H}^{\operatorname{dim}_{\mathscr{H}}(\partial \theta)}(\partial \theta)>0$. Thus, we have that $\mathscr{H}^{d}(\partial \theta)=0$, but

$$
\begin{aligned}
& \mathbb{E}\left[\mathscr{H}^{\operatorname{dim} \mathscr{H}(\partial \Theta)}(\partial \Theta \cap \partial \theta)\right] \\
& =\mathbb{E}\left[\mathscr{H}^{\operatorname{dim} \mathscr{H}(\partial \Theta)}(\partial \Theta \cap \partial \theta) ;\{\omega: \Theta=\theta\}\right] \\
& +\mathbb{E}\left[\mathscr{H}^{\operatorname{dim} \mathscr{H}}(\partial \Theta)(\partial \Theta \cap \partial \theta) ;\{\omega: \Theta \neq \theta\}\right] \\
& \geq \mathbb{E}\left[\mathscr{H}^{\operatorname{dim}_{\mathscr{H}}(\partial \Theta)}(\partial \Theta \cap \partial \theta) ;\{\omega: \Theta=\theta\}\right] \\
& =\mathscr{H}^{\operatorname{dim}_{\mathscr{H}}(\partial \theta)}(\partial \theta)>0 \text {. }
\end{aligned}
$$

Hence, by Definition $9, \Theta$ is not absolutely continuous, that is a contradiction.

The following proposition (see also Capasso and Villa, 2006b) may be useful to verify whether a random set $\Theta$ is absolutely continuous.

Proposition 13 Let $\Theta_{s}$ be a random closed set such that $\operatorname{dim}_{\mathscr{H}} \Theta_{s}=s<d$. Then, for all $A \subset \mathbb{R}^{d}$ such that $\mathscr{H}^{d}(\partial A)=0$,

$\mathbb{E}\left[\mathscr{H}^{s}\left(\Theta_{s} \cap \cdot\right)\right] \ll v^{d} \Longleftrightarrow \mathbb{P}\left(\mathscr{H}^{s}\left(\Theta_{s} \cap \partial A\right)>0\right)=0$.

By Fubini's Theorem it easily follows that for any random closed set $\Theta_{n}$ with Hausdorff dimension $n<d$,

$$
\mathbb{P}\left(x \in \Theta_{n}\right)=0 \quad \text { for } v^{d} \text {-a.e. } x \in \mathbb{R}^{d},
$$

independently of its probability law.

If we suppose now that the expected measure $\mathbb{E}\left[\mu_{\Theta_{n}}\right]$ associated with $\Theta_{n}(n<d)$ is absolutely continuous with respect to $v^{d}$, then the set of points $x \in \mathbb{R}^{d}$ such that $\mathbb{P}\left(x \in \Theta_{n}\right)=0$ becomes "bigger".

Proposition 14 Let $\Theta_{n}$ be a random closed set with Hausdorff dimension $n<d$ and such that $\mathbb{E}\left[\mu_{\Theta_{n}}\right] \ll$ $v^{d}$.

Then for any $\mathscr{H}^{n}$-measurable subset $A$ of $\mathbb{R}^{d}$ with $\mathscr{H}^{n}(A)>0, v^{d}(A)=0$, we have that

$$
\mathbb{P}\left(x \in \Theta_{n}\right)=0 \quad \mathscr{H}^{n} \text {-a.e. } x \in A .
$$

Proof. By contradiction there exists a $\mathscr{H}^{n}$-measurable subset $A$ of $\mathbb{R}^{d}$ with $\mathscr{H}^{n}(A)>0, v^{d}(A)=0$, such that $\mathbb{P}\left(x \in \Theta_{n}\right)>0 \mathscr{H}^{n}$-a.e. $x \in A$.

By Corollary 2.10.48 in Federer (1996) we have that the measure $\mathscr{H}^{n}$ is $\sigma$-finite on $A$, so that we may use Fubini's Theorem. Thus it follows that:

$$
\begin{aligned}
& 0<\int_{A} \mathbb{P}\left(x \in \Theta_{n}\right) \mathscr{H}^{n}(\mathrm{~d} x) \\
& =\int_{A} \int_{\Omega} \mathbf{1}_{\Theta_{n}}(x) \mathbb{P}(\mathrm{d} \omega) \mathscr{H}^{n}(\mathrm{~d} x) \\
& =\int_{\Omega} \int_{A} \mathbf{1}_{\Theta_{n}}(x) \mathscr{H}^{n}(\mathrm{~d} x) \mathbb{P}(\mathrm{d} \omega)=\mathbb{E}\left[\mathscr{H}^{n}\left(\Theta_{n} \cap A\right)\right]
\end{aligned}
$$

But this is in contrast with the assumption $\mathbb{E}\left[\mu_{\Theta_{n}}\right] \ll$ $v^{d}$, since $\mathbb{E}\left[\mathscr{H}^{n}\left(\Theta_{n} \cap A\right)\right]$ should be equal to 0 .

Note that a natural definition related to the absolute continuity of $\mathbb{E}\left[\mu_{\Theta}\right]$ with respect to $v^{d}$ would have been the following:

Definition 15 (Absolute continuity in mean) Let $\Theta$ be a random closed set in $\mathbb{R}^{d}$ such that its associated expected measure $\mathbb{E}\left[\mu_{\Theta}\right]$ is a Radon measure. We say that $\Theta$ is absolutely continuous in mean if the expected measure $\mathbb{E}\left[\mu_{\Theta}\right]$ is absolutely continuous with respect to $v^{d}$.

We may observe that Definition 15 is consistent with the case in which $\Theta$ is a real random variable or a random point in $\mathbb{R}^{d}$, i.e., for $n=$ 0 ; however if $\Theta$ has Hausdorff dimension $d$, then the expected measure $\mathbb{E}\left[\mathscr{H}^{d}(\Theta \cap \cdot)\right]$ is, obviously, always absolutely continuous with respect to the $d$ dimensional Lebesgue measure $v^{d}$, even if $\Theta$ is a deterministic set. Thus, any random closed sets in $\mathscr{R}$ with Hausdorff dimension $d$ is absolutely continuous in mean, but in general it is not absolutely continuous in the strong sense.

Further, there exist some pathological sets of lower dimension which may be either deterministic or discrete and, at the same time, their expected measure is absolutely continuous. For instance, if $\Theta_{n}$ is a random closed set such that, for almost every $\omega \in \Omega, \mathscr{H}^{n}\left(\Theta_{n}(\omega)\right)=0$, then it follows that $\mathbb{E}\left[\mathscr{H}^{n}\left(\Theta_{n}\right)\right]=0$, so that $\mathbb{E}\left[\mu_{\Theta_{n}}\right] \ll v^{d}$, independently of its probability law. Indeed there exist deterministic random closed sets which are absolutely continuous in mean. A simple example is offered by a 2-dimensional Brownian path in a fixed plane in $\mathbb{R}^{3}$ (i.e., consider a realization $\theta_{2}$ of a planar Brownian motion, as a deterministic closed set in a plane in $\mathbb{R}^{3}$ ); it is known that the Hausdorff dimension of $\theta_{2}$ is 2 , but $\mathscr{H}^{2}\left(\theta_{2}\right)=$ 0 . 
Remark 16 If $\Theta$ is a random closed set in $\mathscr{R}$ such that $\operatorname{dim}_{\mathscr{H}}(\Theta)=s<d$, then $\partial \Theta=\Theta$; therefore $\mathbb{E}\left[\mu_{\Theta}\right]=$ $\mathbb{E}\left[\mu_{\partial \Theta}\right]$ and, by definition, it follows that there is no distinction between absolute continuity strong and in mean.

Note that, under usual assumptions in literature (see, e.g., Beneš and Rataj (2004); Stoyan et al. (1995)), a fibre process belong to the class $\mathscr{R}$.

\section{STOCHASTIC BIRTH-AND- GROWTH PROCESSES}

In this section we wish to analyze the case in which a random closed set $\Theta$ may depend upon time as, for example, when it models the evolution due to a growth process, so that we have a geometric random process $\left\{\Theta^{t}, t \in \mathbb{R}_{+}\right\}$, such that for any $t \in \mathbb{R}_{+}$, the random set $\Theta^{t}$ satisfies all the relevant assumptions required in the previous sections. Correspondingly the associated linear functional $\delta_{\Theta^{t}}$ will also be a functional depending on time, and so we need to define partial derivatives of linear functionals depending on more than one variable.

Consider a linear functional $L$ acting on a test space $\mathscr{S}_{k}$ of measurable functions $s$ in $k$ variables; we formally represent it as

$$
(L, s):=\int_{\mathbb{R}^{k}} \phi\left(x_{1}, \ldots, x_{k}\right) s\left(x_{1}, \ldots, x_{k}\right) \mathrm{d}\left(x_{1}, \ldots, x_{k}\right) .
$$

Let us denote by $L_{i}^{h}$ the linear functional defined by

$$
\begin{gathered}
\left(L_{i}^{h}, s\right):= \\
\int_{\mathbb{R}^{k}} \phi\left(x_{1}, \ldots, x_{i}+h, \ldots, x_{k}\right) s\left(x_{1}, \ldots, x_{k}\right) \mathrm{d}\left(x_{1}, \ldots, x_{k}\right) .
\end{gathered}
$$

We define the weak partial derivative of the functional $L$ with respect to the variable $x_{i}$ as follows (see also Gelfand and Shilov, 1964, p. 20).

Definition 17 We say that a linear functional $L$ on the space $\mathscr{S}_{k}$, admits a weak partial derivative with respect to $x_{i}$, denoted by $\frac{\partial}{\partial x_{i}} L$, if and only if $\frac{\partial}{\partial x_{i}} L$ is a linear functional on the same space $\mathscr{S}_{k}$ and $\left\{\frac{L_{i}^{h}-L}{h}\right\}$ weakly* converges to $\frac{\partial}{\partial x_{i}} L$, i.e.,

$$
\lim _{h \rightarrow 0}\left(\frac{L_{i}^{h}-L}{h}, s\right)=\left(\frac{\partial}{\partial x_{i}} L, s\right) \quad \text { for all } s \in \mathscr{S}_{k} .
$$

Let us consider, as an example, the case in which $\left\{\Theta^{t}\right\}_{t}$ is given by a birth-and-growth process (see Fig. 6). A birth-and-growth process is made of two main ingredients; a birth process modelled as a marked point process, and a growth process. As far as the birthand-growth process is concerned, consider a Borel set $E \subseteq \mathbb{R}^{d}, d \geq 2$, endowed with its Borel $\sigma$-algebra $\mathscr{B}_{E}$. A marked point process $N$ on $\mathbb{R}_{+}$, with marks in $E$, is a point process on $\mathbb{R}_{+} \times E$ with the property that the marginal process $\left\{N(B \times E): B \in \mathscr{B}_{\mathbb{R}_{+}}\right\}$is itself a point process. So, it is defined as a random measure given by

$$
N=\sum_{n=1}^{\infty} \varepsilon_{T_{n}, X_{n}}
$$

where

- $T_{n}$ is an $\mathbb{R}_{+}$-valued random variable representing the time of birth of the $n$-th nucleus,

- $\quad X_{n}$ is an $E$-valued random variable representing the spatial location of the nucleus born at time $T_{n}$,

- $\varepsilon_{t, x}$ is the Dirac measure on $\mathscr{B}_{\mathbb{R}_{+}} \times \mathscr{B}_{E}$ such that for any $t_{1}<t_{2}$ and $A \in \mathscr{B}_{E}$,

$$
\varepsilon_{t, x}\left(\left[t_{1}, t_{2}\right] \times A\right)= \begin{cases}1 & \text { if } t \in\left[t_{1}, t_{2}\right], x \in A, \\ 0 & \text { otherwise. }\end{cases}
$$
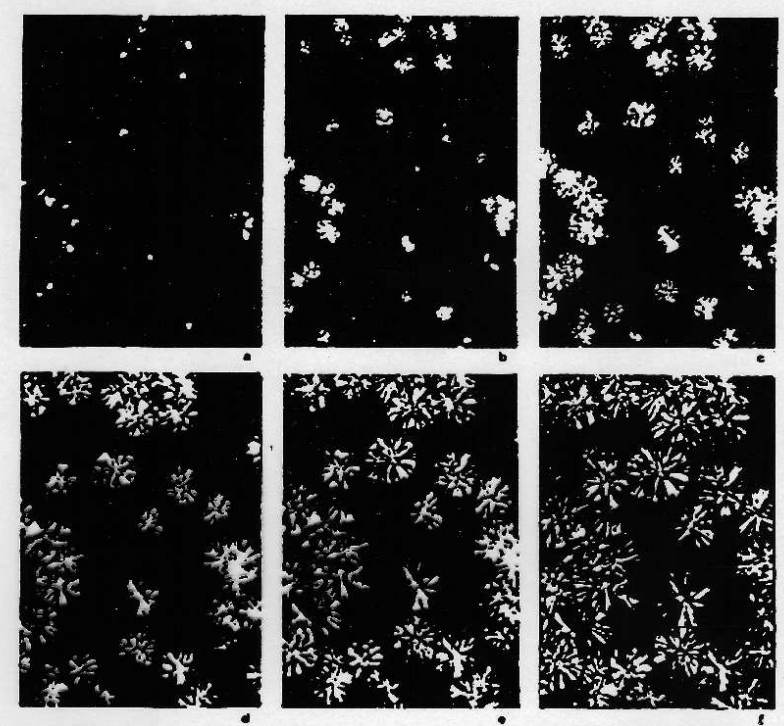

Fig. 6. Time evolution (from top left to bottom right) of a birth-and-growth process: a real polymer crystallization process. Reproduced by permission of BASELL Polyolefins, "G. Natta" Research Centre, Ferrara, Italy.

Hence, in particular, for any $B \in \mathscr{B}_{\mathbb{R}_{+}}$and $A \in \mathscr{B}_{E}$ bounded, we have

$$
N(B \times A)=\#\left\{T_{n} \in B, X_{n} \in A\right\}<\infty,
$$


i.e., it is the (random) number of germs born in the region $A$, during time $B$.

It is well known (Brémaud, 1981; Last and Brandt, 1995) that, under general conditions, a marked point process is characterized by its compensator (or stochastic intensity), say $v(\mathrm{~d} t \times \mathrm{d} x)$, with respect to the internal history $\mathscr{F}_{t}$ of the process. Besides, if $\tilde{v}(\mathrm{~d} t)$ is the compensator of the marginal process, there exists a stochastic kernel $k$ from $\Omega \times \mathbb{R}_{+}$to $E$ such that

$$
v(\mathrm{~d} t \times \mathrm{d} x)=k(t, \mathrm{~d} x) \tilde{v}(\mathrm{~d} t) .
$$

Its expected value $\Lambda(\mathrm{d} t \times \mathrm{d} x):=\mathbb{E}(v(\mathrm{~d} t \times \mathrm{d} x))$ is the so called intensity measure of the process. It is possible to factorize $\Lambda$ in the following way (Last and Brandt, 1995):

$$
\Lambda(\mathrm{d} t \times \mathrm{d} x)=\tilde{\Lambda}(\mathrm{d} t) Q(t, \mathrm{~d} x),
$$

where $\tilde{\Lambda}$ is the intensity measure of the marginal process and, $\forall t \in \mathbb{R}_{+}, Q(t, \cdot)$ is a probability measure on $E$, called the mark distribution at time $t$.

We assume that the nucleation process $N$ is such that the marginal process is simple (i.e., $N(\mathrm{~d} t \times E) \leq 1$ for every infinitesimal time interval $\mathrm{d} t$ ), and so the mark distribution $Q(t, A)$ represents the probability that a nucleus belongs to $A$, given that it is born during $[t, t+\mathrm{d} t)$.

In many cases we have volume crystallization, i.e., the random set $\Theta^{t}$ describing the crystallized region at time $t$ is of the same dimension $d$ as the physical space; in this case it is usually assumed that further nuclei cannot be born in an already crystallized zone. When we want to emphasize this, we have

$$
\begin{aligned}
v(\mathrm{~d} t \times \mathrm{d} x) & =k(t, \mathrm{~d} x) \tilde{v}(\mathrm{~d} t) \\
& =k_{0}(t, \mathrm{~d} x) \tilde{v}_{0}(\mathrm{~d} t)\left(1-\mathbf{1}_{\Theta^{t-}}(x)\right)
\end{aligned}
$$

where $v_{0}(\mathrm{~d} t \times \mathrm{d} x)=k_{0}(t, \mathrm{~d} x) \tilde{v}_{0}(\mathrm{~d} t)$ is the compensator of the process $N_{0}$, called the free-process, in which nuclei can be born anywhere (see also Capasso and Villa, 2005). Accordingly, we denote by $\Lambda_{0}$ the intensity measure of $N_{0}$.

In order to complete the definition of the birth-andgrowth process we need to add a growth process for each individual grain $\Theta_{t_{0}}^{t}\left(x_{0}\right)$ born at some time $t_{0}$, at some location $x_{0}$. Let $\Theta_{T_{n}}^{t}\left(X_{n}\right)$ be the random closed set obtained as the evolution up to time $t>T_{n}$ of the germ born at time $T_{n}$ in $X_{n}$, according to some growth model; this will be the grain associated with the $\operatorname{germ}\left(T_{n}, X_{n}\right)$. In other words, if $T_{n}=s$ and $X_{n}=x$, then $\Theta_{s}^{t}(x)$ is the crystal born at time $s$ and point $x$ and grown up to time $t$.
Definition 18 We call birth-and-growth process the family $\left\{\Theta^{t}\right\}_{t}$ of random closed sets given by

$$
\Theta^{t}=\bigcup_{n: T_{n} \leq t} \Theta_{T_{n}}^{t}\left(X_{n}\right), \quad t \in \mathbb{R}_{+} .
$$

On the other hand an angiogenic process can be modelled as a birth-and-growth process at dimension 1 ; i.e., as a random fibre system (Beneš and Rataj, 2004). Now the marked counting process modelling the birth processes, call it $M$, refers to the offspring of a new capillary from an already existing vessel, i.e., from a point $x$ belonging to an infinitesimal element $\mathrm{d} l$ of the stochastic fibre process $\Theta^{t-}$, so that the branching rate should be of the form

$$
\mu(\mathrm{d} t \times \mathrm{d} l)=\beta(t, \mathrm{~d} l) \tilde{v}(\mathrm{~d} t) \mathbf{1}_{\Theta^{t-}}(x),
$$

where $\beta$ is a suitable (possibly deterministic) kernel. This shows the dependence of the branching rate upon the existing stochastic fibre system $\Theta^{t-}$, and the fact that the point of birth has to belong to its infinitesimal element $\mathrm{d} l$.

With reference to a volume growth process $\left\{\Theta^{t}\right\}_{t}$, a quantity of interest is the random variable $\tau(x)$, representing the time of capture of a given point $x \in E$, i.e.,

$$
\left\{x \in \Theta^{t}\right\}=\{\tau(x) \leq t\},
$$

and correspondingly the well known hazard function $h(\cdot, x)$ associated with point $x$ defined as

$$
h(t, x):=\lim _{\Delta t \downarrow 0} \frac{\mathbb{P}\left(x \in \Theta^{t+\Delta t} \mid x \notin \Theta^{t}\right)}{\Delta t} .
$$

More in general, especially when referring to a fibre process or a fibre system, it is more interesting to study the (random) hitting time $\tau(K)$ of a nontrivial compact $K \subset E$ by $\Theta^{t}$. The associated survival function is then given by

$$
S_{K}(t):=\mathbb{P}(\tau(K)>t)=\mathbb{P}\left(\Theta^{t} \cap K=\emptyset\right)=1-T_{\Theta^{t}}(K),
$$

which shows the direct relationship with the hitting functional $T_{\Theta^{t}}$, which is known to characterize the probability law of the random set $\Theta^{t}$.

Correspondingly a hazard function $h(K, t)$ can be defined as the hitting rate of the process $\Theta^{t}$, i.e.,

$$
h(K, t)=\lim _{\Delta t \rightarrow 0} \frac{\mathbb{P}\left(\Theta^{t+\Delta t} \cap K \neq \emptyset \mid \Theta^{t} \cap K=\emptyset\right)}{\Delta t} .
$$




\section{MODELS OF VOLUME GROWTH}

Models of volume growth have been studied more extensively, since the pioneering work by Kolmogorov (1937). Here we present a theory which extends to the space inhomogeneous case previous results known in literature (Møller, 1992; Capasso, 2003); unfortunately nontrivial mathematical problems arise in connection with angiogenic fibre models that, to our knowledge, remain still unsolved.

For the volume growth case we assume here the normal growth model (see, e.g., Burger (2002)), according to which at $\mathscr{H}^{d-1}$-almost every point of the actual grain surface at time $t$ (i.e., at $\mathscr{H}^{d-1}$ almost every $\left.x \in \partial \Theta_{T_{n}}^{t}\left(X_{n}\right)\right)$, growth occurs with a given strictly positive normal velocity

$$
v(t, x)=G(t, x) n(t, x),
$$

where $G(t, x)$ is a given deterministic strictly positive "growth" field, and $n(t, x)$ is the unit outer normal at point $x \in \partial \Theta_{T_{0}}^{t}\left(X_{0}\right)$. We assume that

$$
0<g_{0} \leq G(t, x) \leq G_{0}<\infty \quad \forall(t, x) \in \mathbb{R}_{+} \times \mathbb{R}^{d},
$$

for some $g_{0}, G_{0} \in \mathbb{R}$, and $G(t, x)$ is sufficient regular (in particular $G$ has to be (globally) Lipschitz-continuous on $\mathbb{R}_{+} \times \mathbb{R}^{d}$ ) such that the evolution problem given by (9) for the growth front $\partial \Theta_{t_{0}}^{t}(x)$, with the initial condition that at the birth time $t_{0}$ the initial germ born in $x_{0}$ is described by a spherical ball of infinitesimal radius centered at $x_{0}$, is well posed. (See, e.g.,Burger, 2002; the case of regularity of $G$ deriving from its coupling with a deterministic underlying field has been analyzed in Su and Burger (2006).)

We may assume that the process $\left\{\Theta^{t}\right\}_{t}$ is such that:

1. for any $t \in \mathbb{R}_{+}$, and any $s>0 ; \partial \Theta^{t} \subset \operatorname{int} \Theta^{t+s}$;

2. for any $t \in \mathbb{R}_{+}, \Theta^{t}$ is a $d$-regular random closed set in $\mathbb{R}^{d}$, and $\partial \Theta^{t}$ is a $(d-1)$-regular random closed set;

3. $\tau(x)$ is a continuous random variable with probability density function $p_{\tau(x)}$.

For the assumptions 1. and 2. we refer to Barles et al. (1993) (see also Burger, 2002). Assumption 3. strongly depends on the intensity of the marked point process of birth-and-growth together with sufficient regularity of the growth rate. For instance, as shown in Capasso and Villa (2005), we know that, if $Q(s, \cdot)$ is absolutely continuous with respect to $v^{d}$ for a.e. $s \in \mathbb{R}_{+}$, then $\tau(x)$ is a continuous random variable.

In particular, from the relation between $\tau(x)$ and the survival function $S_{x}$ of a point $x$

$$
S_{x}(t)=\mathbb{P}(\tau(x)>t),
$$

we may claim that $\tau(x)$ admits a density $p_{\tau(x)}$ if $S_{x}$ is differentiable with respect to $t$. In Capasso and Villa (2005) it is shown that there is no distinction between $N$ and $N_{0}$ in computing the survival function, and expressions for $S_{x}$ are given for a general class of birthand-growth processes. In the particular case in which the free-process $N_{0}$ is a marked Poisson process, it follows that

$$
S_{x}(t)=e^{-\Lambda_{0}(\mathscr{C}(t, x))},
$$

where $\Lambda_{0}$ is the intensity measure of $N_{0}$, and $\mathscr{C}(t, x)$ is the so-called causal cone of point $x$ at time $t$, defined as the space-time region in which at least one nucleation has to take place so that the point $x$ is covered by grains at time $t$ :

$$
\mathscr{C}(t, x):=\left\{(s, y) \in[0, t] \times E: x \in \Theta_{s}^{t}(y)\right\} .
$$

In Burger et al. (2006) it has been proven that, under the assumptions on $G$ introduced above, if the marked Poisson process $N_{0}$ has free intensity $\alpha(t, x)$, then $\Lambda_{0}(\mathscr{C}(t, x))$ is continuously differentiable with respect to $t$ and

$$
\begin{aligned}
& \frac{\partial}{\partial t} \Lambda_{0}(\mathscr{C}(t, x))=h(t, x)= \\
& G(t, x) \int_{0}^{t} \int_{\mathbb{R}^{d}} K\left(t_{0}, x_{0} ; t, x\right) \alpha\left(t_{0}, x_{0}\right) \mathrm{d} x_{0} \mathrm{~d} t_{0}
\end{aligned}
$$

with

$$
K\left(t_{0}, x_{0} ; t, x\right):=\int_{\left\{z \in \mathbb{R}^{d} \mid \tau\left(t_{0}, x_{0} ; z\right)=t\right\}} \delta(z-x) \mathrm{d} \sigma(z)
$$

Here $\delta$ is the usual Dirac function, $\operatorname{d} \sigma(z)$ is a $(d-$ $1)$-dimensional surface element, and $\tau\left(t_{0}, x_{0} ; z\right)$ is the solution of the eikonal problem

$$
\begin{gathered}
\left|\frac{\partial \tau}{\partial x_{0}}\left(t_{0}, x_{0}, x\right)\right|=\frac{1}{G\left(t_{0}, x_{0}\right)} \frac{\partial \tau}{\partial t_{0}}\left(t_{0}, x_{0}, x\right) \\
\left|\frac{\partial \tau}{\partial x}\left(t_{0}, x_{0}, x\right)\right|=\frac{1}{G\left(\tau\left(t_{0}, x_{0}, x\right), x\right)},
\end{gathered}
$$

subject to suitable boundary and initial conditions

Further we assume that $G(t, x)$ is sufficiently regular so that, at almost any time $t>0$, the following holds (see Capasso and Villa (2006d))

$$
\lim _{r \rightarrow 0} \frac{\mathbb{E}\left[\mathscr{H}^{d}\left(\Theta_{\oplus r}^{t} \backslash \Theta^{t} \cap A\right)\right]}{r}=\mathbb{E}\left[\mathscr{H}^{d-1}\left(\partial \Theta^{t} \cap A\right)\right]
$$

for any bounded $A \in \mathscr{B}_{\mathbb{R}^{d}}$ such that $\mathbb{P}\left(\mathscr{H}^{d-1}\left(\partial \Theta^{t} \cap\right.\right.$ $\partial A)>0)=0$. 
Here we have denoted by $\Theta_{\oplus r}^{t}$ the parallel set of $\Theta^{t}$ at distance $r \geq 0$, i.e., the Minkowski addition of $\Theta^{t}$ with the ball $B_{r}(0)$ :

$$
\Theta_{\oplus r}^{t}:=\Theta^{t} \oplus B_{r}(0)=\left\{x \in \mathbb{R}^{d}: \operatorname{dist}\left(x, \Theta^{t}\right) \leq r\right\} .
$$

Observe that, in terms of weak* convergence of linear functionals, (10) may be rephrased as

$$
\lim _{r \rightarrow 0} \frac{\mathbb{E}\left[\delta_{\Theta_{\oplus r}^{t}}\right](x)-\mathbb{E}\left[\delta_{\Theta^{t}}\right](x)}{r}=\mathbb{E}\left[\delta_{\partial \Theta^{t}}\right](x) .
$$

As a consequence of assumptions 1. and 2. we have that (see Capasso and Villa, 2006d)

$$
\frac{\partial}{\partial t} \delta_{\Theta^{t}}(x)=\delta_{\tau(x)}(t)
$$

as a functional on $C_{c}\left(\mathbb{R}_{+} \times \mathbb{R}^{d}, \mathbb{R}\right)$, i.e.,

$$
\begin{aligned}
& \int_{\mathbb{R}_{+} \times \mathbb{R}^{d}} f(t, x) \frac{\partial}{\partial t} \delta_{\Theta^{t}}(x) \mathrm{d} t \mathrm{~d} x= \\
& =\int_{\mathbb{R}_{+} \times \mathbb{R}^{d}} f(t, x) \delta_{\tau(x)}(t) \mathrm{d} t \mathrm{~d} x=\int_{\mathbb{R}^{d}} f(\tau(x), x) \mathrm{d} x
\end{aligned}
$$

for any test function $f \in C_{c}\left(\mathbb{R}_{+} \times \mathbb{R}^{d}, \mathbb{R}\right)$.

By the assumption 3. and Remark 6, it follows:

$\mathbb{E}\left[\frac{\partial}{\partial t} \delta_{\Theta^{t}}\right](x)=p_{\tau(x)}(t)=\frac{\partial}{\partial t} \mathbb{P}\left(x \in \Theta^{t}\right)=\frac{\partial}{\partial t} \mathbb{E}\left[\delta_{\Theta^{t}}\right](x)$.

As a consequence an evolution equation for the mean density $\mathbb{E}\left[\delta_{\Theta^{t}}\right](x)$ has been obtained in terms of $G(t, x)$ and the mean density of the boundary of $\Theta^{t}$, as follows (Capasso and Villa, 2006d).

Proposition 19 Under the above assumption on the growth model, let $G$ be sufficiently regular so that, for any $t \in \mathbb{R}_{+}$,

$$
\lim _{r \rightarrow 0} \frac{\mathbb{E}\left[\mathscr{H}^{d}\left(\Theta_{\oplus r}^{t} \backslash \Theta^{t} \cap A\right)\right]}{r}=\mathbb{E}\left[\mathscr{H}^{d-1}\left(\partial \Theta^{t} \cap A\right)\right],
$$

for any $A \in \mathscr{B}_{\mathbb{R}^{d}}$ such that $\mathbb{E}\left[\mathscr{H}^{d-1}\left(\partial \Theta^{t} \cap \partial A\right)\right]=0$.

If the time of capture $\tau(x)$ is a continuous random variable with density $p_{\tau(x)}$, the following evolution equation holds for the mean density $\mathbb{E}\left[\delta_{\Theta^{t}}\right](x)$ :

$$
\frac{\partial}{\partial t} \mathbb{E}\left[\delta_{\Theta^{t}}\right](x)=G(t, x) \mathbb{E}\left[\delta_{\partial \Theta^{t}}\right](x),
$$

to be taken, as usual, in weak form, i.e., for any bounded $A \in \mathscr{B}_{\mathbb{R}^{d}}$ such that $\mathbb{E}\left[\mu_{\partial \Theta^{t}}(\partial A)\right]=0$

$$
\begin{aligned}
& \int_{A} \mathbb{E}\left[\delta_{\Theta^{t}}\right](x) \mathrm{d} x= \\
& \int_{A} \mathbb{E}\left[\delta_{\Theta^{t_{0}}}\right](x) \mathrm{d} x+\int_{t_{0}}^{t} \int_{A} G(x, s) \mathbb{E}\left[\delta_{\partial \Theta^{s}}\right](x) \mathrm{d} x \mathrm{~d} s .
\end{aligned}
$$

Note that when for a.e. $\omega \in \Omega$ the evolution of the realization $\Theta^{t}(\omega)$ can be described by the following (weak) equation (e.g., Sokolowski and Zolesio, 1992; Barles et al., 1993; Lorenz, 2001; Burger, 2002):

$$
\frac{\partial}{\partial t} \delta_{\Theta^{t}}(x)=G(t, x) \delta_{\partial \Theta^{t}}(x)
$$

then equation (11) can be formally obtained by taking the expected value in (12), by the linearity properties of the expectation and since $G$ is a deterministic function.

Remark 20 By Proposition 19 and the equality $p_{\tau(x)}(t)=\frac{\partial}{\partial t} \mathbb{E}\left[\delta_{\Theta^{t}}\right](x)$, it follows that $\mathbb{E}\left[\delta_{\partial \Theta^{t}}\right]$ is the classical Radon-Nikodym derivative of $\mathbb{E}\left[\mu_{\partial \Theta^{t}}\right]$. Assumption 2 guarantees that $\Theta^{t}$ belongs to the class $\mathscr{R}$ for any $t \in \mathbb{R}_{+}$. Thus, by Definition 9, we may claim that $\Theta^{t}$ is an absolutely continuous random closed set.

\section{APPROXIMATION OF MEAN DENSITIES}

In many real applications, several problems are related to the estimation of the local mean density $\mathbb{E}\left[\delta_{\Theta_{n}}\right]$ of a lower dimensional random closed set such as a fibre process of dimension $n=1$ in a space of dimension $d>1$ (see, e.g., Stoyan et al., 1995 and Beneš and Rataj, 2004).

For facing the problem of the zero $v^{2}$-measure for points or lines in $\mathbb{R}^{2}$ it is natural to make use of a 2D box approximation of points or lines. As a matter of fact, a computer graphic representation of them is anyway provided in terms of pixels, which can only offer a 2-D box approximation of points in $\mathbb{R}^{2}$.

This is the motivation of this section, which aims to suggest unbiased estimators for densities of random sets of lower dimensions in a given $d$-dimensional space (Ambrosio et al., 2006).

Given a random closed set $\Theta_{n}$ with Hausdorff dimension $n$, we consider the enlarged set $\Theta_{n_{\oplus r}}$, which is now of dimension $d$, and hence of nontrivial $v^{d}$ measure.

Observe that $\mathbb{P}\left(x \in \Theta_{n_{\oplus r}}\right)=T_{\Theta_{n}}\left(B_{r}(x)\right)$. 
Proposition 21 (Ambrosio et al., 2006) Let $\Theta_{n}$ be a random closed set with Hausdorff dimension $n$, and $A \in \mathscr{B}_{\mathbb{R}^{d}}$ such that $\mathbb{P}\left(\mathscr{H}^{n}\left(\Theta_{n} \cap \partial A\right)>0\right)=0$. If

$$
\lim _{r \rightarrow 0} \frac{\mathbb{E}\left[v ^ { d } \left(\Theta_{\left.\left.n_{\oplus r} \cap A\right)\right]}\right.\right.}{b_{d-n} r^{d-n}}=\mathbb{E}\left[\mathscr{H}^{n}\left(\Theta_{n} \cap A\right)\right]
$$

then

$$
\mathbb{E}\left[\mathscr{H}^{n}\left(\Theta_{n} \cap A\right)\right]=\lim _{r \rightarrow 0} \int_{A} \frac{T_{\Theta_{n}}\left(B_{r}(x)\right)}{b_{d-n} r^{d-n}} \mathrm{~d} x
$$

Sufficient conditions for (13) have been given in Ambrosio et al. (2006).

As a consequence of the proposition above, if we denote by $\mu^{\oplus r}$ the measure on $\mathscr{B}_{\mathbb{R}^{d}}$ defined by

$$
\mu^{\oplus r}(A):=\int_{A} \frac{T_{\Theta_{n}}\left(B_{r}(x)\right)}{b_{d-n} r^{d-n}} \mathrm{~d} x
$$

then it follows that $\mu^{\oplus r}$ weakly* converges to $\mathbb{E}\left[\mu_{\Theta_{n}}\right]$.

For every fixed $r>0$, the measure $\mu^{\oplus r}$ is absolutely continuous with respect to the $d$ dimensional Lebesgue measure with density

$$
\delta_{n}^{\oplus r}(x):=\frac{T_{\Theta_{n}}\left(B_{r}(x)\right)}{b_{d-n} r^{d-n}} .
$$

Such a function defines a linear functional, say $\delta_{n}^{\oplus r}$, associated with the measure $\mu^{\oplus r}$ as follows

$$
\left(\delta_{n}^{\oplus r}, f\right):=\int_{\mathbb{R}^{d}} f(x) \mu^{\oplus r}(\mathrm{~d} x) .
$$

Note that many kinds of random closed sets satisfy the proposition above, like fibre processes, line and segment processes, Boolean models, etc. (see Ambrosio et al., 2006). As a consequence, estimating the probability that the random set $\Theta_{n}$ intersects the ball $B_{r}(x)$ may suggest (global) estimators of $\mathbb{E}\left[\mu_{\Theta_{n}}\right]$, and possibly (local) estimators of the mean density $\mathbb{E}\left[\delta_{\Theta_{n}}\right]$ (see, e.g., Beneš and Rataj, 2004).

If $\Theta_{n}$ is absolutely continuous, then there exists an integrable function $\lambda_{\Theta_{n}}$ (the Radon-Nikodym derivative) such that, for all $A \in \mathscr{B}_{\mathbb{R}^{d}}$,

$$
\mathbb{E}\left[\mathscr{H}^{n}\left(\Theta_{n} \cap A\right)\right]=\int_{A} \lambda_{\Theta_{n}}(x) \mathrm{d} x .
$$

So, in this case, we have that

$$
\lim _{r \rightarrow 0} \int_{A} \frac{T_{\Theta_{n}}\left(B_{r}(x)\right)}{b_{d-n} r^{d-n}} \mathrm{~d} x=\int_{A} \lambda_{\Theta_{n}}(x) \mathrm{d} x .
$$

If $\Theta_{n}$ is a stationary random closed set, then $\delta_{n}^{\oplus r}(x)$ is independent of $x$ and the expected measure $\mathbb{E}\left[\mu_{\Theta_{n}}\right]$ is motion invariant, i.e., it is absolutely continuous with density $\lambda_{\Theta_{n}}(x)=\bar{\lambda} \in \mathbb{R}_{+}$for $v^{d}$-a.e. $x \in \mathbb{R}^{d}$. It follows that

$$
\lim _{r \rightarrow 0} \int_{A} \frac{T_{\Theta_{n}}\left(B_{r}(x)\right)}{b_{d-n} r^{d-n}} \mathrm{~d} x=\lim _{r \rightarrow 0} \frac{T_{\Theta_{n}}\left(B_{r}(0)\right)}{b_{d-n} r^{d-n}} v^{d}(A),
$$

and

$$
\int_{A} \lambda(x) \mathrm{d} x=\bar{\lambda} v^{d}(A)
$$

and so, by (14),

$$
\lim _{r \rightarrow 0} \frac{T_{\Theta_{n}}\left(B_{r}(0)\right)}{b_{d-n} r^{d-n}}=\bar{\lambda}
$$

Remark 22 When it is possible to exchange limit and integral in (14), by Proposition 21 we may claim that

$$
\lim _{r \rightarrow 0} \frac{T_{\Theta_{n}}\left(B_{r}(x)\right)}{b_{d-n} r^{d-n}}=\lambda_{\Theta_{n}}(x) \quad v^{d} \text {-a.e. } x \in \mathbb{R}^{d} .
$$

In the particular case $n=d$, we know that the measure $\mathbb{E}\left[\mu_{\Theta_{d}}\right]$ is always absolutely continuous with density $\lambda_{\Theta_{d}}(x)=\mathbb{P}\left(x \in \Theta_{d}\right)$. We may notice that $\delta_{d}^{\oplus r}=$ $T_{\Theta_{n}}\left(B_{r}(x)\right)$ and by Monotone Convergence Theorem we can exchange limit and integral, and so we have, as expected,

$$
\lim _{r \rightarrow 0} T_{\Theta_{d}}\left(B_{r}(x)\right)=\mathbb{P}\left(x \in \Theta_{d}\right)=\lambda_{\Theta_{d}}(x) .
$$

Further, for $n=0$, if $\Theta_{0}=X$ is a random point in $\mathbb{R}^{d}$, we have $\mathbb{E}\left[\mathscr{H}^{0}(X \cap \cdot)\right]=\mathbb{P}(X \in \cdot)$. So, if $X$ is absolutely continuous with probability density function $f$, we know that $\mathbb{E}\left[\mu_{X}\right]=\mathbb{P}_{X}$ is absolutely continuous with density $f$. In this case it can be shown that (13) holds, so that the sequence $\left\{\boldsymbol{\delta}^{\oplus r}(x)\right\}$ converges to $f(x)$, as expected, which leads to the usual histogram estimation of $f(x)$ (Ambrosio et al., 2006).

Example As an additional example of applicability of the results above, let us consider the case in which $\Theta_{n}$ is given by a random union of absolutely continuous random closed sets of dimension $n<d$ :

$$
\Theta_{n}=\bigcup_{i=1}^{\Phi} E_{i},
$$

where $\Phi$ is a nonnegative discrete random variable with $\mathbb{E}[\Phi]<\infty$, and the $E_{i}$ 's are IID as $E$ and independent of $\Phi$. Then it follows that (Ambrosio et al., 2006)

$$
\lim _{r \rightarrow 0} \frac{T_{\Theta_{n}}\left(B_{r}(x)\right)}{b_{d-n} r^{d-n}}=\mathbb{E}[\Phi] \lim _{r \rightarrow 0} \frac{T_{E}\left(B_{r}(x)\right)}{b_{d-n} r^{d-n}},
$$


provided that at least one of the two limits exists.

As a consequence, whenever it is possible to exchange limit and integral in (14), and so in particular when $E$ is a stationary random closed set (which implies $\Theta_{n}$ stationary as well), we have

$$
\lambda_{\Theta_{n}}(x)=\mathbb{E}[\Phi] \lim _{r \rightarrow 0} \frac{T_{E}\left(B_{r}(x)\right)}{b_{d-n} r^{d-n}}=\mathbb{E}[\Phi] \lambda_{E}(x),
$$

where $\lambda_{\Theta_{n}}$ and $\lambda_{E}$ are the Radon-Nikodym derivatives of $\mu_{\Theta_{n}}$ and $\mu_{E}$, respectively.

The above model may be used as a preliminary one for angiogenesis, but also for the earthworm burrow system in a soil (Beneš and Rataj, 2004, p. 73).

\section{ACKNOWLEDGEMENTS}

It is a pleasure to acknowledge the contribution of L. Ambrosio in Pisa, M. Burger in Linz and A. Micheletti in Milan in the development of joint research projects relevant for this presentation. Special thanks are due to Professor E. Dejana and her group for figures 2 and 4, and BASELL Polyolefins, "G. Natta" Research Centre, Ferrara, Italy (in particular Dr. S. Mazzullo) for figures 1, and 6. Discussions with D. Jeulin evidenced the $\mathrm{PhD}$ thesis of Matheron as a first attempt to deal with random distributions.

\section{REFERENCES}

Ambrosio L, Capasso V, Villa E (2006). On the approximation of geometric densities of random closed sets. RICAM Report 14/2006, Linz.

Ambrosio L, Fusco N, Pallara D (2000). Functions of Bounded Variation and Free Discontinuity Problems. Oxford: Clarendon Press.

Anderson ARA (2003). Effects of cell adhesion on solid tumour geometry. In: Sekimura $\mathrm{T}$ et al., eds. Morphogenesis and Pattern Formation in Biological Systems. Tokyo: Springer-Verlag.

Araujo A, Giné E (1980). The Central Limit Theorem for Real and Banach Valued Random Variables. New York: John Wiley \& Sons.

Avrami A (1939). Kinetic of phase change. Part I. J Chem Phys 7:1103-12.

Baddeley AJ, Molchanov IS (1997). On the expected measure of a random set. In: Proceedings of the International Symposium on Advances in Theory and Applications of Random Sets (Fontainebleau, 1996). River Edge, NJ: World Sci. Publishing, 1:3-20.

Barles G, Soner HM, Souganidis PE (1993). Front propagation and phase-field theory. SIAM J Contr Optim 31:439-69.
Beneš V, Rataj J (2004). Stochastic Geometry. Dordrecht: Kluwer.

Bosq D (2000). Linear Processes in Function Spaces. Theory and Applications. Lecture Notes in Statistics 149. New York: Springer-Verlag.

Brémaud P (1981). Point Processes and Queues, Martingale Dynamics. New York: Springer-Verlag.

Burger M (2002). Growth fronts of first-order HamiltonJacobi equations. SFB Report 02-8. Linz: J. Kepler University.

Burger M, Capasso V, Pizzocchero L (2006). Mesoscale averaging of nucleation and growth models. Multiscale Model Sim 5:564-92.

Burger M, Capasso V, Salani C (2002). Modelling multidimensional crystallization of polymers in interaction with heat transfer. Nonlinear Anal-Real 3:139-160.

Capasso V, ed (2003). Mathematical Modelling for Polymer Processing. Polymerization, Crystallization, Manufacturing. Mathematics in Industry Vol 2. Heidelberg: Springer Verlag.

Capasso V, Micheletti A (2005). Stochastic geometry and related statistical problems in biomedicine. In: Quarteroni A et al, eds. Complex Systems in Biomedicine. Milano: Springer.

Capasso V, Villa E (2005). Survival functions and contact distribution functions for inhomogeneous stochastic geometric marked point processes. Stoch Anal Appl 23:79-96.

Capasso V, Villa E (2006a) On the evolution equations of mean geometric densities for a class of space and time inhomogeneous stochastic birth-and-growth processes. In: Weil W. ed. Stochastic Geometry. Lecture Notes in Mathematics - CIME subseries 1892. Heidelberg: Springer, 267-81.

Capasso V, Villa E (2006b). On the continuity and absolute continuity of random closed sets. Stoch Anal Appl 24:381-397.

Capasso V, Villa E (2006c). Some remarks on the continuity of random closed sets. In: Lechnerová R, Saxl I, Beneš $\mathrm{V}$ eds. Proceedings of the International Conference in Stereology, Spatial Statistics and Stochastic Geometry. Prague: UCMP, 69-74.

Capasso V, Villa E (2006d). On the geometric densities of random closed sets. RICAM Report 13/2006, Linz.

Capasso V, Villa E (2007). On the generalized geometric densities of random closed sets. An application to growth processes. In: Aletti $\mathrm{G}$ et al., eds. Math Everywhere. Deterministic and stochastic modelling in Biomedicine, Economics and Industry. Heidelberg: Springer-Verlag. 
Carmeliet P, Jain RK (2000). Angiogenesis in cancer and other diseases. Nature 407:249-57.

Chaplain MAJ, Anderson ARA (1999). Modelling the growth and form of capillary networks. In: Chaplain MAJ et al., eds. On Growth and Form. Spatio-temporal Pattern Formation in Biology. Chichester:John Wiley \& Sons.

Corada M, Zanetta L, Orsenigo F, Breviario F, Lampugnani MG, Bernasconi S, Liao F, Hicklin DJ, Bohlen P, Dejana E (2002). A monoclonal antibody to vascular endothehal-cadherin inhibits tumor angiogenesis without side effects on endothelial permeability. Blood 100:905-11.

Crosby CV, Fleming PA, Argraves WS, Corada M, Zanetta L, Dejana E, Drake CJ (2005). VE-cadherin is not required for the formation of nascent blood vessels but acts to prevent their disassembly. Blood 105:2771-6.

Falconer KJ (1985). The Geometry of Fractal Sets. Cambridge: Cambridge University Press.

Federer H (1996). Geometric Measure Theory. Berlin: Spriger.

Gelfand IM, Shilov GE (1964). Generalized Functions. Properties and Operations. New York: Academic Press.

Hahn U, Micheletti A, Pohlink R, Stoyan D, Wendrock $\mathrm{H}$ (1999). Stereological Analysis and Modeling of Gradient Structures. J Microsc 195:113-24.

Johnson WA, Mehl RF (1939). Reaction kinetics in processes of nucleation and growth. Trans AIMME 135:416-58.

Jones DS (1982). The Theory of Generalised Functions. Cambridge: Cambridge University Press.

Kolmogorov AN (1937). On the statistical theory of the crystallization of metals. Bull Acad Sci USSR Math Ser 1:355-9.
Kolmogorov AN, Fomin SV (1970). Introductory Real Analysis. Englewood Cliffs, NJ: Prentice-Hall.

Last G, Brandt A (1995). Marked Point Processes on the Real Line. The Dynamic Approach. New York: Springer.

Lorenz T (2001). Set valued maps for image segmentation. Comput Visual Sci 4:41-57.

Matheron G (1965). Les Variables Regionalisées et leur Estimation. Paris: Masson et Cie.

Matheron G (1975). Random sets and integral geometry. New York: John Wiley \& Sons.

Møller J (1992). Random Johnson-Mehl tessellations. Adv Appl Probab 24:814-44.

Møller J (1994). Lectures on Random Voronoi Tessellations. Lecture Notes in Statistics 87. New York, Berlin, Heidelberg: Springer-Verlag,

Sokolowski J, Zolesio J-P (1992). Introduction to Shape Optimization. Shape Sensitivity Analysis. Berlin: Springer-Verlag.

Stoyan D, Kendall WS, Mecke J (1995). Stochastic Geometry and its Application. New York: John Wiley \& Sons.

Su B, Burger M (2006). Weak solutions of a polymer crystal growth model. CAM-Report 06-40, UCLA.

Ubukata T (2003). Computer modelling of microscopic features of molluscan shells. In: Sekimura, T et al., eds. Morphogenesis and Pattern Formation in Biological Systems. Tokyo: Springer-Verlag.

Vladimirov VS (1979). Generalized Functions in Mathematical Physics. Moscow: Mir Publishers.

Zahle M (1982). Random processes of Hausdorff rectifiable closed sets. Math Nachr 108:49-72. 